\title{
Agua y pobreza en Santiago de Chile. Morfología de la inequidad en la distribución del consumo domiciliario de agua potable
}

Gustavo Durán. Flacso (Facultad Latinoamericana de Ciencias Sociales-Sede Ecuador), Departamento de Asuntos Públicos, Quito, Ecuador.

RESUMEN | En la trayectoria temporal de la relación entre agua y pobreza en Santiago de Chile, el "acceso" como indicador ha sufrido, para los estudios urbanos, una suerte de desgaste, y en la actualidad no representa un dato significativo para medir las condiciones de inequidad en esta sociedad urbana. De otra parte, el "consumo" sobresale como un indicador con mayor capacidad de revelar las circunstancias de vida de los pobres en esa ciudad, que si bien les ofrece posibilidades de inserción en términos de acceso a las redes físicas del sistema, los excluye a través de mecanismos de mercado, como un sistema tarifario en permanente proceso de crecimiento y el desbalance de este respecto del ingreso familiar. Este proceso sostenido de contracción del consumo domiciliario de agua potable que se viene registrando a partir de la privatización, se produce en el marco de un claro proceso de "mercantilización” (Winpenny, 1994, p.110) de un bien básico, como lo es el agua en la ciudad.

PALABRAS CLAVE | pobreza, servicios urbanos, consumo.

ABSTRACT | Based on the story of the relationship between water and poverty in Santiago de Chile, the "access" as an indicator has experienced, for urban studies, a kind of wear and tear, and today does not represent a significant data to measure the inequality in this urban society. On the other hand, "consumption" stands out as an indicator with a greater capacity to reveal the circumstances of life of the poor people in that city, because even if it offers integration possibilities in terms of physical network access system, it excludes them by market mechanisms, such as a system that is permanently increasing fares and has no relation to family income. This contraction of domestic consumption of drinking water that has been recorded since the privatization of the service is produced in the context of a clear process of "commodification" (Winpenny, 1994, p.110) of a good, such as water in the city.

KEYWORDS | poverty, urban services, consumption. 


\section{Introducción}

El proceso modernizador que el sector sanitario chileno inició desde el comienzo de la dictadura, se hizo bajo un esquema que pretendía reorganizar el Estado y la sociedad en un proyecto neoliberal unificador (Bauer, 2002). Uno de sus grandes logros ha sido que los indicadores de acceso a las redes de agua potable y alcantarillado en las zonas urbanas, y concretamente en la Región Metropolitana de Santiago (RMS), han estado muy cerca de la cobertura universal desde hace más de dos décadas.

La modernización del sector sanitario iniciada en 1977 (Figueroa, 2004) se da en un contexto de reducción del tamaño del Estado y de fortalecimiento del rol de los actores privados en los sectores estratégicos de la planificación de las ciudades. A mediados de los noventa (1994) tuvo lugar la transacción definitiva, y las mayores empresas sanitarias del país fueron transferidas a grandes grupos económicos. La Empresa Municipal de Obras Sanitarias (EMOs), que lideraba el mercado de agua en Santiago de Chile, fue adquirida por un consorcio hispano-francés, "Aguas", y pasó a llamarse "Aguas Andinas".

En resumen, un sector que ya era eficiente desde el punto de vista empresarial (Morandé \& Doña, 1997), logró continuar esa senda y a través de la inversión privada fue respondiendo a nuevos desafíos, el más grande de los cuales era (y continúa siendo) elevar el volumen de tratamiento de aguas servidas.

Al privatizar el servicio, el sistema tarifario fue diseńado para cubrir tres aspectos: los costos reales del servicio, las utilidades de los propietarios y los planes de desarrollo de las empresas. La evidencia encontrada señala que, desde la privatización de la emos, las tarifas han tenido hasta la fecha un proceso de crecimiento continuo y que, por otra parte, los niveles de consumo domiciliario de unidades físicas en el contexto urbano de Santiago han bajado también de forma progresiva (Valenzuela \& Jouravlev, 2007).

Este artículo busca comprender el resultado de todo ese proceso, y pretende responder la siguiente pregunta de investigación: ¿cómo se inscribe en el espacio urbano del Gran Santiago este fenómeno de contracción del consumo domiciliario de agua potable, y qué relación se puede establecer con los procesos de fragmentación y segregación urbana por los que atraviesa la ciudad?

La hipótesis que se va a demostrar es que la contracción del consumo domiciliario de agua potable, que vienen registrando los sectores de más bajos ingresos a partir del proceso de privatización, responde a la configuración de un sistema tarifario en permanente proceso de encarecimiento y a su desequilibrio respecto a un ingreso familiar precario, un proceso que agudiza los desequilibrios territoriales preexistentes en la ciudad de Santiago.

Históricamente, la relación entre agua y pobreza tiene profundas raíces espaciales; es decir, el "lugar" donde los pobres habitan generalmente ha tenido dificultades de acceso al agua potable en alguna de las fases de provisión de este servicio. En el caso concreto de Santiago ha sido así, y con mayor fuerza debido al rol del mercado en la política del suelo que se viene implementando desde el gobierno militar hasta la actualidad (Tironi, 2003). 
Pflieger (2008) pone de relieve la importante incidencia que ha tenido la evolución del acceso al agua potable en la definición del tejido urbano de la ciudad de Santiago y en sus reconocidas realidades de segregación y fragmentación. Sin embargo, la universalización del servicio de agua potable -hoy la ciudad tiene cerca de un $100 \%$ de cobertura- ha desdibujado este vínculo entre agua y pobreza.

Hace más de quince años, Rodríguez (1994) constató un nuevo ámbito de la relación agua y pobreza, pues aparentemente, con la universalización de las redes de infraestructura de agua potable, emergió un nuevo desafío en términos de superación de la pobreza urbana: la equidad en el consumo. Su planteamiento es que el diseño del nuevo modelo de gestión del sector de agua potable es funcional a la lógica del mercado, pero está desarticulado de las verdaderas capacidades de la demanda.

Rodríguez (1994) reconstruye la relación entre agua y pobreza en Santiago y relocaliza el debate en torno a la noción de "brecha de consumo", la cual subraya el hecho de presentar amplios diferenciales en las unidades físicas de agua consumidas en territorios relativamente pequeños. Tal noción tiene en la actualidad plena vigencia, pues cuestiona la capacidad regulatoria del mercado del agua urbana por parte del Estado, en el contexto de la privatización neoliberal.

A partir de esta nueva comprensión de la relación entre agua y pobreza, con una amplia base espacial y enfocada ya no desde las redes sino de la capacidad de consumo, es importante anotar, en torno a la reflexión de Swyngedouw (2004), que en el fondo de este debate persiste la discusión sobre la ciudadanía y el derecho a la ciudad. Es en este contexto teórico donde se inscribe este artículo: en la mercantilización o commodification (Swyngedouw, 2004) del agua urbana y en cómo dicho procesos es una muestra de la racionalidad que domina el desarrollo urbano actual, una lógica que afecta la capacidad de quienes habitan la ciudad en para constituirse en ciudadanos.

Bakker (2001) complementa a Swyngedouw, en términos de que esa mercantilización del agua en la ciudad se da aun con una presencia parcial del Estado, pues no se requiere una retirada total de este para hacer efectiva la llamada commodification. El caso chileno pareciera que responde a ese patrón, en el cual el Estado no desaparece, sino que se refuncionaliza y va creando un marco institucional que genera las reglas del juego necesarias para hacer del agua en la ciudad una mercancía. Así, las características que Bakker describe como los actuales principios ordenadores del sector sanitario bajo la racionalidad neoliberal, como la eficiencia económica y la base tecnocrática en la toma de decisiones, son los ejes de los procesos modernizadores que condujeron las reformas del sector desde 1977 hasta la actualidad.

Con anterioridad a la toma del control por parte de los actores privados en el sector agua ya existían deficiencias en conectividad y calidad, las cuales se traducían -en términos de consumo- en bajos precios y mayor accesibilidad vía consumo, sobre todo para los sectores de bajos ingresos. Pero a partir de los procesos de modernización dados en la fase neoliberal, tal y como Zaki y Amin (2009) lo afirman, esta relación se invierte: se perfeccionan los sistemas en términos de expansión y universalización de las redes y la calidad del servicio se eleva de forma integral; pero ello tiene un efecto directo en la conformación del precio para el consumidor final.

El objetivo de este trabajo es aproximarse a esos procesos de transformación de las condiciones de pobreza y exclusión en una ciudad como Santiago de Chile, 
donde -según afirman los estudios- la reducción progresiva en el consumo de unidades físicas de agua es aparentemente uno de los principales efectos de la privatización del sector sanitario iniciada a finales de la década de los setenta (Valenzuela \& Jouravlev, 2007).

Para lograr tal objetivo, es importante comprender que el consumo de agua en la ciudad está condicionado por determinantes tanto socioeconómicos como territoriales, lo que hace de él una estructura compleja. Kayaga, Calvert y Sansom (2003) afirman que para comprender bien el comportamiento del consumo de agua en los hogares, es preciso descomponerlo en determinantes que, al modificarse alguna de ellas, de la misma forma modifica el resultado final. Zhanga y Brownb (2005) distinguen al respecto variables políticas, tecnológicas, ambientales y demográficas.

Es a partir de los anteriores postulados que para este artículo se han definido dos categorías o variables que van a ayudar a comprender mejor los comportamientos del consumo de agua potable en Santiago en el contexto de la privatización: el sistema tarifario, y los ingresos y presupuesto familiar.

La primera determinante del consumo es el sistema tarifario. Se trata de un acuerdo técnico-político establecido por el modelo de gobernanza del agua en el país/ciudad, y que en el contexto de la privatización en Chile surge de una negociación entre la empresa privada y el Estado regulador. En este aspecto, Kayaga, Calvert y Sansom (2003) establecen una relación inversa entre las tarifas y el consumo.

Davis (2005) hace un recorrido extenso por la historia de la participación de los actores privados en el sector sanitario, de donde surge claramente que, a medida que crece su liderazgo, las tarifas se incrementan, lo que ha tenido un efecto claro en las características del consumo. El caso chileno no ha sido la excepción. Es por eso que, a la hora de descomponer la estructura interna del consumo domiciliario, este trabajo se va a iniciar estableciendo la relación existente entre el registro del consumo y el sistema tarifario.

La segunda determinante del consumo comprende los ingresos y presupuesto familiar. Al respecto, Kayaga, Calvert y Sansom (2003) establecen una relación positiva entre el ingreso de los hogares y el consumo de agua que registran. En el área de operación de la empresa prestadora del servicio, tales ingresos están directamente asociados a la actividad económica de los jefes de hogar.

Esta determinante resulta crucial para esta investigación, pues su objeto de investigación es la pobreza y, en particular, las nuevas formas en que se manifiesta. Las teorías acerca de la nueva pobreza urbana (Wilson, 1998) coinciden en que el elemento central donde se cimientan las condiciones actuales de exclusión en la ciudad se explican, ante todo, por el estado de desarticulación y precarización del mercado laboral, sobre todo en los sectores medios y bajos de la economía.

Estas dos determinantes teóricas serán operativizadas a través de un análisis espacial, el cual se dividirá en dos partes, y será la variable "escala territorial" el eje estructurante de esta investigación. El estudio se va a focalizar en un intento por desagregar los promedios de consumo domiciliario, partiendo de los grandes territorios operacionales de las empresas sanitarias, para llegar a analizar el consumo comunal. La intención es, por un lado, medir los niveles de desigualdad socioterritorial en el consumo de agua potable; y, por otro, aproximarse a los patrones 
espaciales de localización de aquellos clientes que están consumiendo cada vez menos agua potable en Santiago de Chile.

La primera parte del análisis, de carácter introductorio, busca conocer la composición empresarial y territorial del mercado sanitario en la Región Metropolitana de Santiago. En esta aproximación, la unidad espacial de análisis estará compuesta por los territorios operacionales de las siete principales concesionarias sanitarias que proveen del servicio a casi la totalidad de los clientes urbanos de la región.

Esta parte introductoria del análisis espacial se desarrollará a una escala urbanoregional. Sin embargo, la información consolidada en los reportes de gestión del órgano regulador estatal, la Superintendencia de Servicios Sanitarios (sISs), provee datos agregados por empresa concesionaria, y es por eso que este estudio parte por reconocer la división espacial de estas empresas, pues sus contratos de concesión están ligados fundamentalmente a un territorio de operaciones, tanto en agua potable como en alcantarillado.

En la segunda parte del análisis se dará un paso más en este análisis espacial en términos de escala. Se pasará, entonces, de una escala urbano-regional a una escala urbana consolidada y se seleccionará el más grande y heterogéneo de los territorios operacionales estudiados en la parte anterior, el correspondiente a la concesión de Aguas Andinas (Gran Santiago). La unidad espacial de análisis estará constituida por las comunas o la parte de ellas que estén al interior de su área de abastecimiento; esto con el propósito de establecer la localización y estratificación de los clientes de la mayor empresa concesionaria de agua potable de Chile. Como base, se tomará información relativa a los meses de febrero de 2009, 2007 y 2005, considerando que durante el verano se registran los niveles de consumo más altos del año.

\section{Composición empresarial y territorial del mercado sanitario, y el consumo de agua potable en la Región Metropolitana de Santiago}

En la RMs operan principalmente siete empresas que controlan el mercado de agua potable urbana y, según la clasificación de la siss, una de ellas, Aguas Andinas, corresponde a las denominadas como empresas "mayores"; otra de ellas, sMAPA (Servicio Municipal de Agua Potable y Alcantarillo, la empresa pública de la comuna de Maipú), está dentro de las llamadas empresas "medianas"; y las cinco restantes, Aguas Cordillera, Aguas Los Domínicos, Aguas Manquehue, Servicomunal y Servilampa, son del grupo de las empresas clasificadas como "menores". El criterio de esta clasificación corresponde, ante todo, al tamaño de la concesión, en términos geográficos y de población atendida (siss, 2007).

Para empezar, entonces, se presentará de forma descriptiva la localización y datos generales de estas siete principales concesionarias sanitarias; a continuación, se establecerá el volumen total de agua potable que factura cada una de ellas, para poder estimar un promedio de consumo por cliente; al final se entregarán algunos datos acerca del consumo per cápita en la RMs. El objetivo de esta serie de análisis es graficar, de forma breve pero contundente, esa noción de "brecha de consumo" que Rodríguez (1994, p. 33) ha estudiado en el pasado, y que hoy este estudio quiere actualizar. En general, se trabajará con datos de 2007; sin embargo, fue importante, en la medida 
en que se tuvo acceso a información, establecer series de tiempo, para verificar ese supuesto inicial de que el consumo a escala urbano-regional viene registrando una contracción sostenida en el periodo iniciado con la privatización del servicio.

\section{Localización y datos generales de las siete principales concesionarias sanitarias}

La Región Metropolitana de Santiago, ubicada en la zona central de la República de Chile, tiene una extensión de $15.394,70 \mathrm{~km}^{2}$. El total del área concesionada por la Superintendencia de Servicios Sanitarios de Chile (sIss) en la Región Metropolitana es de $847,41 \mathrm{~km}^{2}$, y en su interior habitaban aproximadamente 6.396 .148 personas en 2007. El territorio que está por fuera de estas concesiones corresponde, en términos del modelo de gestión del servicio de agua potable y alcantarillado, a un esquema diferente al utilizado para aglomeraciones urbanas, y que es llamado por su sigla APR (Agua Potable Rural). El área concesionada corresponde a los territorios operacionales de las siete principales empresas sanitarias, en cuyo contrato de concesión están perfectamente establecidas las coordenadas geográficas con las cuales se determina su área de cobertura (Figura 1).

FIGURA I | Territorios operacionales de las principales concesionarias sanitarias en la Región Metropolitana de Santiago

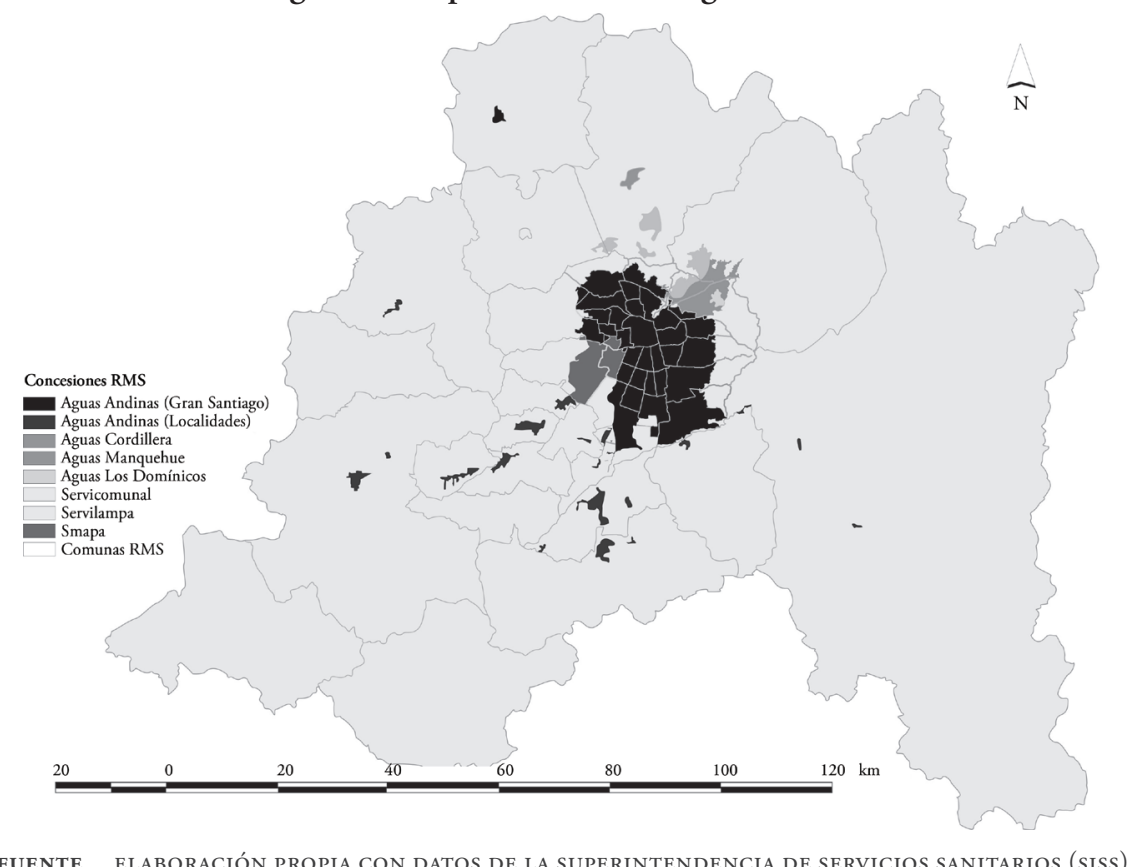

Según la Superintendencia de Servicios Sanitario de Chile, las áreas de concesión de las siete empresas que controlaron la casi totalidad del mercado urbano en la Región Metropolitana de Santiago (RMS) durante el año 2007 fueron, según su tamaño y características, como sigue: 
- Tres empresas menores -Aguas Cordillera, Aguas Los Domínicos y Aguas Manquehue- de naturaleza privada y con un área de concesión de $77,24 \mathrm{~km}^{2}, 12,04$ $\mathrm{km}^{2}$ y $41,24 \mathrm{~km}^{2}$, respectivamente. Estas empresas menores, que tradicionalmente atendían al sector de altos ingresos localizado en el noreste de la ciudad de Santiago, posteriormente atendieron los nuevos desarrollos inmobiliarios del sector norte, igualmente dirigidos a la población de altos ingresos. En suma, estas tres empresas prestaron el servicio de agua potable durante el ańo 2007 a una población urbana de 337.946 habitantes de la RMS.

- Las dos restantes empresas menores, Servicomunal y Servilampa, cuentan con un área de concesión de $8,21 \mathrm{~km}^{2}$ y $3,85 \mathrm{~km}^{2}$, respectivamente, las cuales son el producto del aumento endógeno de población de pequeños asentamientos al norte de la ciudad (en las comunas de Colina y Lampa), y que, luego de superar los umbrales frecuentemente trazados para un sistema de agua potable de gestión comunitaria o rural, se constituyeron en empresa concesionaria y atendieron en 2007 a una población urbana de 89.023 habitantes.

- Una empresa de categoría mediana, llamada Servicio Municipal de Agua Potable y Alcantarillado de Maipú (sMAPA), de propiedad de la Municipalidad de Maipú y con un territorio de operaciones de $75,23 \mathrm{~km}^{2}$. En 2007 prestó el servicio a 687.863 habitantes de las comunas de Maipú, Cerrillos y Estación Central.

- La mayor empresa sanitaria concesionaria del país, Aguas Andinas, una compañía privada que posee un área de concesión de $629,58 \mathrm{~km}^{2}$, con una cobertura de 5.241.316 habitantes, y un territorio distribuido en dos secciones. La primera es llamada Localidades, y comprende pequeños territorios correspondientes a poblados satélite de la ciudad de Santiago; la extensión de esta área de concesión es de 91,91 km². El otro grupo de la concesión de Aguas Andinas es llamado Gran Santiago; su área de cobertura corresponde parcialmente a la antigua compañía estatal llamada Empresa Metropolitana de Obras Sanitarias (EMOs), y para el año 2007 tenía una extensión de 537,66 km².

De las siete empresas mencionadas, cuatro de ellas -Aguas Andinas, Aguas Cordillera, Aguas Los Domínicos y Aguas Manquehue- forman parte del "Grupo Aguas", que es el más grande concesionario de servicios sanitarios en el país, y que solamente para la Región Metropolitana controla el 88,8\% de los clientes.

\section{Consumo total de agua potable en la RMs}

El tema de las áreas de concesión se vincula al eje central de este estudio, esto es, el consumo residencial de agua potable. Durante 2007, al interior de las siete áreas de concesión se facturó un total de 534 millones de $\mathrm{m}^{3}$ de agua potable.

A primera vista, existe una coherencia aparente entre tamańo del área de la concesión y volumen de unidades físicas de agua facturadas. No se observan casos sobresalientes en los cuales una concesión demasiado pequeña supere el volumen de agua facturada a una concesión más extensa. Una razón plausible para ello puede ser que efectivamente las concesiones, al no incluir zonas rurales, tienen entre sí una relación área-consumo, aparentemente proporcional en las comunas urbanizadas de Santiago. Es evidente el control de mercado que tiene el Grupo Aguas respecto 
de los demás en el contexto de la Región Metropolitana, pues del total de metros cúbicos de agua consumida facturados en 2007, este grupo suministró el 89,75\%.

Otro elemento para anotar es que, en 2006, el volumen de agua facturada en la región fue de 528 millones de $\mathrm{m}^{3}$. Este dato demuestra que en este periodo (20062007), el consumo global de la Región Metropolitana subió en un 0,96\%. Sin embargo, este aumento respondió sobre todo a la incorporación de nuevos clientes, pues el consumo promedio por cliente disminuyó.

\section{Facturación promedio por cliente}

En términos generales, Aguas Manquehue tiene un volumen mucho más bajo de unidades físicas totales facturadas para el año 2007 que el de Aguas Andinas: tan solo 8 millones de $\mathrm{m}^{3}$, respecto a los 405 millones de $\mathrm{m}^{3}$ de la última. Sin embargo, si se considera el agua potable suministrada por cliente, Aguas Manquehue resulta ser una empresa que provee casi cinco veces más que Aguas Andinas, debido a que la población que abastece es mucho más pequeña (tendría que multiplicarse por 188 para igualar la cantidad de población que atiende Aguas Andinas) (Figura 2).

FIGURA 2 Dotación diaria de agua potable por persona en la Región Metropolitana de Santiago por empresa concesionaria

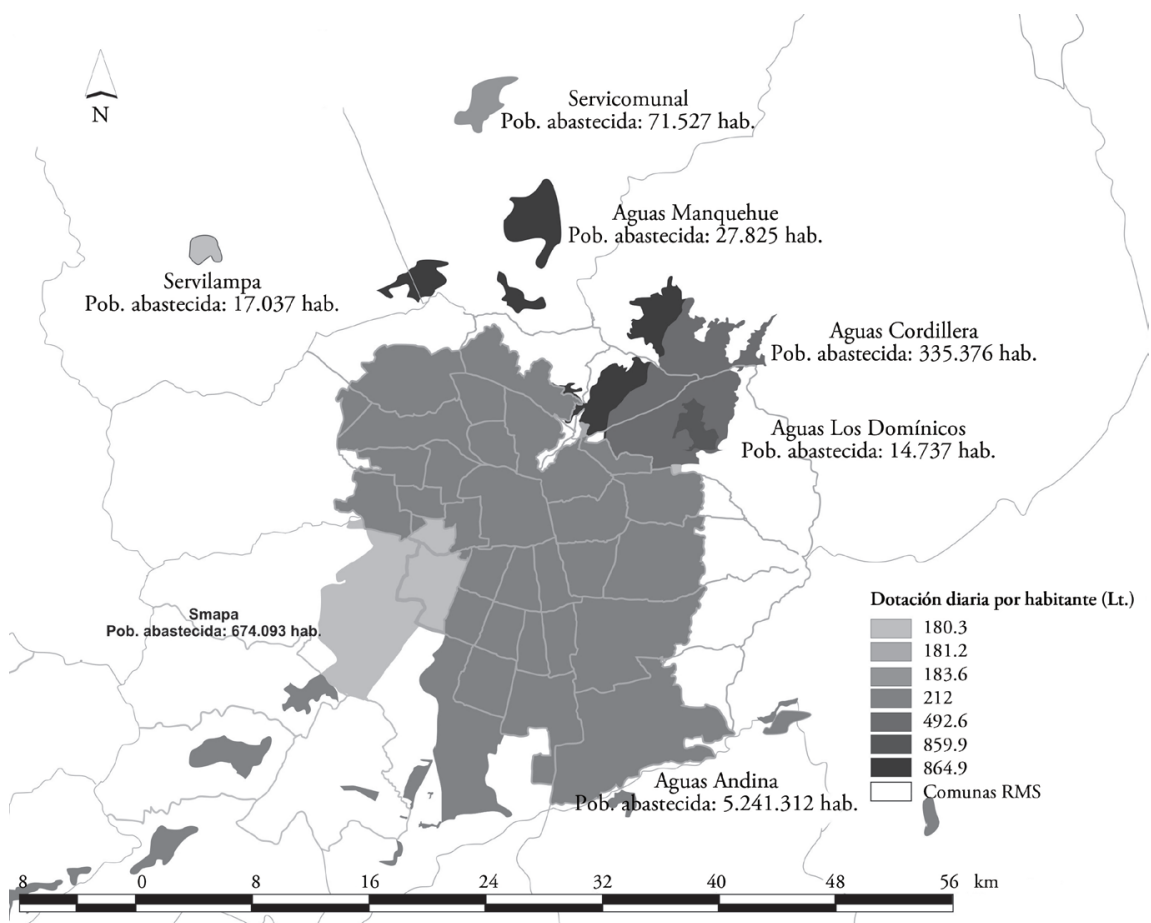

FUENTE ELABORACIÓN PROPIA CON DATOS DE LA SUPERINTENDENCIA DE SERVICIOS SANITARIOS (SISS) 
En cambio, cuando se compara Aguas Andinas con SMAPA, la relación entre la población atendida y la facturación en miles de metros cúbicos es proporcional: registra un consumo promedio por cliente relativamente parecido, $21 \mathrm{~m}^{3}$ y $23 \mathrm{~m}^{3}$, respectivamente. Ambas empresas, al estar dentro del rango medio y alto de empresas de la RMS, hacen que el promedio de la región en términos del consumo por cliente total sea bastante similar al promedio de ellas, pues el tamańo de las otras cinco empresas es marginal. Se puede agregar un dato adicional, y es cómo se ha comportado ese consumo promedio en los últimos años a escala empresarial.

A partir de 1999, cuando el proceso de privatización ya estuvo bien consolidado, la Superintendencia de Servicios Sanitarios comenzó a emitir informes estandarizados. El primero de ellos, relativo a los consumos altos, señala que Aguas Manquehue y Aguas Los Domínicos han registrado siempre el más alto consumo. Sin embargo, la primera de esas empresas ha tenido un comportamiento más variable en el tiempo, con una elevación en el consumo de hasta casi los $140 \mathrm{~m}^{3}$ por cliente, y luego una caída significativa, para cerrar el periodo con un consumo de sobre $120 \mathrm{~m}^{3}$ por cliente al mes. Aguas Los Domínicos, por su parte, ha tenido en el conjunto de las siete empresas la caída más significativa en este periodo: de más de $130 \mathrm{~m}^{3}$, cayó por debajo de $110 \mathrm{~m}^{3}$ por cliente al mes. En solitario, Aguas Cordillera también registra una caída de aproximadamente $5 \mathrm{~m}^{3}$, para terminar el periodo en casi $40 \mathrm{~m}^{3}$ por cliente al mes.

En el grupo de empresas menores, que atienden hogares de bajos consumos, pero que -como se dijo anteriormente- tienen la mayor área de concesión y atienden a la mayor cantidad de población, Aguas Andinas, SMAPA y Servicomunal también muestran una caída leve, estando las tres en el promedio de la región, cercano a 21 $\mathrm{m}^{3}$ por cliente al mes. Servilampa registra una subida desde su creación en 2005, y termina con el mismo promedio de las anteriores.

\section{Promedios de la dotación diaria de agua potable por persona}

Aunque las empresas que atienden los sectores de altos ingresos han sido las que más han disminuido su consumo por cliente en términos porcentuales, todas lo han hecho al terminar 2007. Si se estima el consumo per cápita por comuna, la situación sigue siendo la misma en términos de consumo diferencial de agua potable en la Región Metropolitana. Así, si la empresa concesionaria Aguas Manquehue, que atiende a 27.825 personas, destina aproximadamente 864,9 litros por día por habitante, la empresa smapa de la Municipalidad de Maipú, que sirve a 674.093 personas, provee 180,3 litros diarios por habitante.

Aguas Andinas tiene un promedio un poco más alto que el de SMAPA: registra un total de 212 litros diarios para cada uno de sus 5.241.312 habitantes atendidos. Sin embargo, y aunque a este nivel empresarial se puede apreciar las grandes diferencias en el consumo regional, surgen preguntas como: ‘todas las personas que habitan dentro de la concesión de Aguas Andinas reciben 212 litros por día? ¿¿de qué forma se distribuye ese consumo en cada comuna? Pues si las empresas que atienden exclusivamente a los sectores de altos ingresos registran mayores consumos, es posible suponer que al interior de un área concesionada socioeconómicamente muy heterogénea, como la de Aguas Andinas, se deben presentar consumos igualmente 
diferenciales en su interior. Con el propósito de responder algunas de estas preguntas, se continuará con el análisis en la siguiente sección.

\section{Localización y estratificación de los clientes de la mayor empresa concesionaria de agua potable de Chile}

Esta tercera parte se iniciará, entonces, con un análisis de la tipología de clientes -industriales, comerciales y residenciales- que atiende Aguas Andinas, pues es necesario estimar el grado de importancia que tiene el consumo de los hogares en el contexto general del mercado de agua. Se logrará conocer la distribución comunal de los distintos tipos de clientes, para luego concentrase en el foco de este estudio, el consumo residencial. Ello porque, independientemente del peso económico que tienen los demás clientes, los hogares son cuantitativamente definitivos en la definición de estos sistemas urbanos de agua potable.

El propósito de analizar la distribución de los clientes según tipología al interior de esta concesión es desagregar los promedios empresariales, los cuales son óptimos desde todo punto de vista, y buscar si existen o no diferencias significativas con los otros tipos de clientes, a partir de la estimación de promedios comunales de consumo de metros cúbicos de agua y alcantarillado por cliente, y según cuántos de ellos acceden al subsidio estatal. Para terminar esta parte del análisis, se calculará, para las comunas del área geográfica en estudio, los promedios de los montos que pagan los clientes por disfrutar del servicio de parte de la empresa sanitaria, y cuánto de ese monto es asumido por la política social del Estado a través de un subsidio.

Para este estudio, en general, se tomaron los consumos realizados en los meses de febrero de 2009, 2007 y 2005, los cuales, por ser verano, constituyen la época del año en que el consumo llega a sus niveles más altos.

Totales y promedios de consumo de agua potable en el territorio operacional de agua potable de Aguas Andinas (Gran Santiago)

En esta sección, se analizará la distribución del consumo por comunas dentro del área de la concesión de Aguas Andinas (Gran Santiago). Para hacerlo, el estudio se dividirá en tres partes. La primera de ellas se concentrará en la composición de la tipología de clientes que tiene cada comuna y su relación con las otras; la segunda, también a una escala comunal, determinará el consumo de unidades físicas de agua potable $\left(\mathrm{m}^{3}\right)$; y la tercera, en estrecha relación con la anterior, explorará en los montos facturados por ese consumo (en moneda nacional).

\section{Clientes}

En este Territorio Operacional de Agua Potable (тод) de Aguas Andinas se concentra la más grande aglomeración urbana de Chile, con un total de 4.276.900 habitantes ${ }^{1}$. Sin embargo, para efectos de este análisis acerca del consumo de agua, el dato al cual se tiene acceso no es por habitante, ni por hogares, sino por cliente. 
Aguas Andinas reportó a la Superintendencia de Servicios Sanitarios para el mes de febrero del año 2009 un total de 1.329.034 clientes. Las comunas de Santiago y Puente Alto son las que lideran la cantidad de clientes adscritos a la concesión, con más de 100.000 conexiones, y se puede observar un grupo considerable de comunas ubicadas en el pericentro suroccidental de la ciudad que tienen menos de 30.000 clientes.

Para profundizar en el análisis, es necesario incorporar el tamaño de la población atendida y ver qué relación tiene con la cantidad de clientes reportados. El objetivo es determinar los extremos de la distribución, según el número de habitantes por cada cliente reportado por Aguas Andinas en cada comuna de su territorio operacional. Desde tal perspectiva, se tiene la siguiente distribución:

- De uno a dos habitantes por cliente, solamente está la comuna de Santiago $(1,68)$. Esto se explica ante todo porque es también la comuna que marca los mayores registros en el número clientes industriales y comerciales, además de ser una comuna con un alto porcentaje de viviendas unipersonales. La amplia oferta inmobiliaria de departamentos de un solo dormitorio en la comuna puede ser reflejo de ello.

- De dos a tres habitantes por cliente está, por un lado, el sector nororiente de altos ingresos (Providencia, Nuñoa, Las Condes, Quilicura), que tiene una densidad poblacional menor y, en casos como Providencia, se ha orientado hacia un desarrollo mixto que combina el uso residencial con el comercial; por otro lado se tiene la comuna de San Miguel, que ha tenido un desarrollo inmobiliario en altura importante recientemente.

- De cuatro a cinco habitantes por cliente están las comunas mayoritariamente ubicadas en el pericentro y periferia surponiente, las cuales se han caracterizado por un menor dinamismo inmobiliario y por no ser en ellas donde mayoritariamente se desarrollan las actividades comerciales de la capital chilena.

El total de la concesión presenta un número de 3,22 habitantes por cliente registrado por Aguas Andinas.

Antes de pasar a analizar los clientes residenciales, que son el objeto de este estudio, se puede dar un rápido vistazo a cómo se distribuyen los clientes industriales y comerciales del área concesionada por Aguas Andinas. La mayor concentración de los clientes industriales se da en las comunas de Santiago Centro (entre 250 y 450 clientes aproximadamente), seguida por las comunas de Quinta Normal, Recoleta, San Joaquín, Macul y San Bernardo, en un rango de 175 a 250 clientes dedicados a la industria. Se observa también una ausencia casi total de clientes industriales en las comunas de la precordillera sur, las cuales, exceptuando la comuna de La Reina, tienen en cada caso menos de diez clientes industriales.

Por otra parte, los datos que registran la localización de clientes comerciales en la concesión señalan que ellos se localizan mayoritariamente en la comuna de Santiago, con más de 16.000 clientes, seguida de cerca por Providencia, con casi de 8.000. Por encima de 2.000 clientes comerciales, se encuentran las comunas de Quinta Normal, Recoleta, Nuñoa, San Miguel, La Florida y Puente Alto. Las actividades comerciales presentan un registro menor de clientes en el extremo norponiente del 
área de concesión, al igual que en comunas situadas al sur de la capital, como La Pintana, La Granja, San Ramón y Lo Espejo.

Como dato adicional, se debe agregar que aunque el número de clientes es bajo en los sectores industrial y comercial comparado con el número de clientes residenciales, la proporción de la demanda de unidades físicas de agua no es igual, pues en el caso de los clientes industriales del territorio operacional de Aguas Andinas, el promedio de consumo para febrero del año 2009 fue de $360,5 \mathrm{~m}^{3}$ por cliente, y en el caso de los clientes comerciales, de $111,9 \mathrm{~m}^{3}$ por cliente.

Ahora bien, siendo el objeto de estudio de este trabajo las transformaciones en las estructuras de consumo domiciliario de agua potable en Santiago de Chile, su foco se concentrará en la información relativa a los clientes residenciales.

Para el mes de febrero del año 2009, se observa cómo la comuna que más clientes residenciales aporta a la concesión de Aguas Andinas es Puente Alto, con casi 150.000 casos. En seguida, destacan las comunas de San Bernardo, La Florida, Santiago y Nuñoa, con un rango que va de 50.000 a 100.000 casos; luego, Quilicura, Pudahuel, Providencia, Peñalolén y La Pintana, que están en el tercer rango, de 35.000 a 50.000 casos.

En el rango medio, de 25.000 a 35.000 clientes, están ubicadas las comunas de Renca, Conchalí, Recoleta, Macul y El Bosque.

En los rangos inferiores de aporte de clientes por comuna, de 20.000 a 25.000 , se encuentra un grupo compuesto por las comunas de Cerro Navia, Lo Prado, Quinta Normal, Las Condes, La Reina, Pedro Aguirre Cerda, San Miguel, San Joaquín, Lo Espejo y La Granja. El resto de las comunas no mencionadas se encuentra por debajo de los 20.000 clientes reportados.

Para un análisis más profundo, se va a contrastar los datos sobre número de clientes con el tamaño promedio de los hogares en cada comuna, información registrada en el Censo de Población y Vivienda de 2002.

Excluyendo a Huechuraba, una comuna caracterizada por una oferta inmobiliaria de baja densidad, generalmente asociada a desarrollos habitacionales unifamiliares con bajo coeficiente de ocupación del suelo, las comunas que destacan entre el grupo de las que registran un promedio mayor de personas por hogar, son aquellas tradicionalmente pobres del sur de la ciudad, como Puente Alto, La Pintana, La Granja, Lo Espejo, El Bosque, San Bernardo; y del occidente, como Renca, Pudahuel y Cerro Navia.

En el otro extremo de la relación, las comunas que menos población registran por hogar son Providencia, Santiago Centro, Las Condes y Nuñoa. Estos cuatro primeros registros representan las comunas donde se ha dado el desarrollo inmobiliario en altura más importante de la ciudad, y son, dentro de la concesión de Aguas Andinas, aquellas que en promedio perciben mayores ingresos.

El resto de comunas de la RMS se distribuye en un rango muy corto, entre 3,42 y 3,77 habitantes por hogar, marcando así el promedio de la ciudad en 3,61. Es importante anotar que cuando se establece este promedio a nivel urbano, sube en 0,39 el promedio anteriormente estudiado de población por clientes, lo cual indica que existe un número no menor de hogares que comparten más de una conexión al servicio. 
Para terminar esta sección dedicada a los clientes de la concesión Aguas Andinas, se verá el promedio de clientes que acceden al subsidio que de forma focalizada otorga el Estado a quienes demuestran incapacidad para pagar el servicio de agua potable. Al respecto, se puede constatar que, en términos generales, el rango del promedio de clientes que recibieron subsidio estatal durante febrero de 2009, oscila entre el 1\% y 25\% del total de los clientes de la concesión de Aguas Andinas. El promedio de toda la concesión está en $8 \%$.

Las comunas que mayor proporción de clientes subsidiados tienen son San Ramón y Cerro Navia, que reciben alrededor del 16\% de los subsidios; y las que menos, en un rango inferior a 1\%, están Santiago, Providencia y Las Condes.

\section{Consumo de unidades físicas}

A partir del análisis previo, relativo a la distribución y composición de los clientes de la concesionaria Aguas Andinas, en que se analizaron -entre otros- elementos como el tipo de clientes para la cantidad de población y el tamaño promedio por hogares, en este apartado se va a examinar el dato fundamental de este estudio: el consumo (Figura 3).

Figura 3 Promedio de consumo de $\mathrm{m}^{3}$ de agua potable por comuna en el territorio operacional de Aguas Andinas (2009)

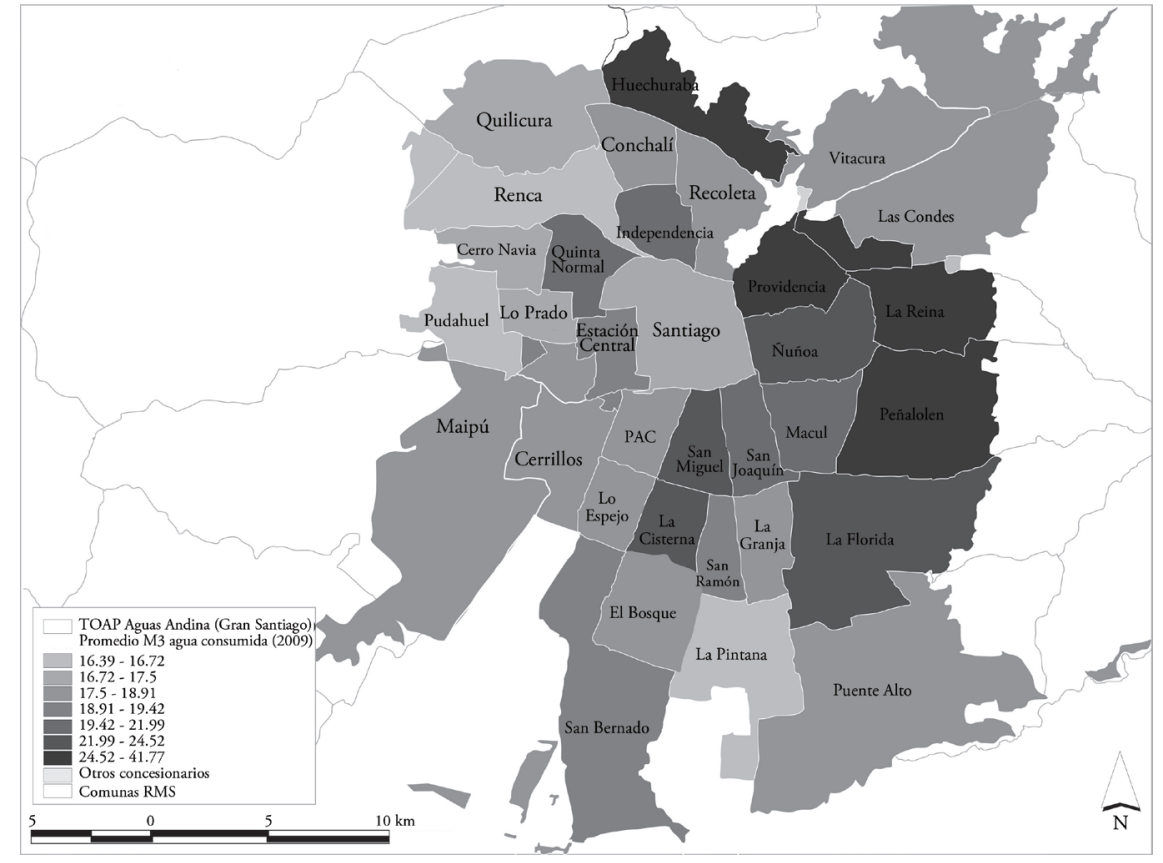

FUENTE ELABORACIÓN PROPIA CON DATOS DE LA SUPERINTENDENCIA DE SERVICIOS SANITARIOS (SISS)

Se va a comenzar, entonces, por un análisis del consumo comunal promedio por cliente, correspondiente al mes de febrero de 2009 (Cuadro 1). 
CUADro I | Promedios comunales de agua potable consumida por cliente de la concesionaria Aguas Andinas, Región Metropolitana de Santiago, febrero 2009, 2007 y 2005, y promedio ingreso autónomo por hogar, 2006

\begin{tabular}{|c|c|c|c|c|}
\hline COMUNA & $\begin{array}{c}\text { PROMEDIO } \\
\left(M^{3}\right) \text { DE AGUA } \\
\text { CONSUMIDA. } \\
\text { FEBRERO } 2009\end{array}$ & $\begin{array}{c}\text { PROMEDIO } \\
\left(\mathbf{M}^{3}\right) \text { DE AGUA } \\
\text { CONSUMIDA. } \\
\text { FEBRERO } 2007\end{array}$ & $\begin{array}{c}\text { PROMEDIO } \\
\left(\mathbf{M}^{3}\right) \text { AGUA } \\
\text { CONSUMIDA. } \\
\text { FEBRERO } 2005\end{array}$ & $\begin{array}{c}\text { INGRESO } \\
\text { AUTÓNOMO } \\
\text { PROMEDIO DEL } \\
\text { HOGAR 2006 } \\
\left(\$ \text { CHILENOS) }\left(^{*}\right)\right.\end{array}$ \\
\hline Pudahuel & 16,39 & 17,86 & 18,36 & 592.626 \\
\hline La Pintana & 16,64 & 15,89 & 16,52 & 403.495 \\
\hline Renca & 16,71 & 18,19 & 18,74 & 516.357 \\
\hline Maipú & 16,72 & 8,30 & 3,42 & 774.457 \\
\hline Santiago & 17,14 & 18,60 & 22,02 & 865.067 \\
\hline Lo Prado & 17,21 & 18,61 & 20,20 & 503.932 \\
\hline Cerro Navia & 17,42 & 19,18 & 20,42 & 408.191 \\
\hline Quilicura & 17,50 & 17,19 & 16,63 & 647.878 \\
\hline Lo Espejo & 18,45 & 17,96 & 19,11 & 414.210 \\
\hline La Granja & 18,49 & 18,25 & 20,40 & 436.865 \\
\hline Puente Alto & 18,68 & 17,43 & 17,82 & 703.863 \\
\hline Pedro Aguirre Cerda & 18,77 & 19,55 & 20,13 & 542.753 \\
\hline El Bosque & 18,79 & 18,53 & 20,13 & 464.439 \\
\hline Recoleta & 18,91 & 19,70 & 22,19 & 463.524 \\
\hline Conchalí & 18,91 & 20,10 & 21,37 & 520.661 \\
\hline San Ramón & 19,03 & 19,32 & 20,36 & 573.482 \\
\hline San Bernardo & 19,09 & 18,01 & 19,50 & 478.831 \\
\hline Estación Central & 19,42 & 20,26 & 21,30 & 648.508 \\
\hline Quinta Normal & 20,40 & 20,79 & 23,48 & 536.445 \\
\hline Independencia & 20,92 & 21,03 & 24,29 & 611.820 \\
\hline San Joaquín & 21,11 & 22,51 & 23,70 & 573.482 \\
\hline Macul & 21,99 & 22,60 & 24,33 & 568.877 \\
\hline La Florida & 22,72 & 21,89 & 23,09 & 900.098 \\
\hline San Miguel & 23,20 & 23,53 & 25,47 & 1.197 .829 \\
\hline Cerrillos & 23,29 & 25,58 & 25,02 & 667.770 \\
\hline La Cisterna & 23,72 & 24,32 & 26,34 & 729.029 \\
\hline Ñuńoa & 24,52 & 26,00 & 28,71 & 1.430 .379 \\
\hline Huechuraba & 28,95 & 30,10 & 33,12 & 687.804 \\
\hline Las Condes & 29,09 & 30,29 & 32,21 & 2.045 .533 \\
\hline Peñalolén & 29,50 & 29,18 & 29,81 & 716.975 \\
\hline Providencia & 30,56 & 33,19 & 36,36 & 1.625 .851 \\
\hline La Reina & 41,77 & 42,05 & 44,38 & 1.275 .726 \\
\hline Promedio total & 21,08 & 21,30 & 22,69 & \\
\hline
\end{tabular}

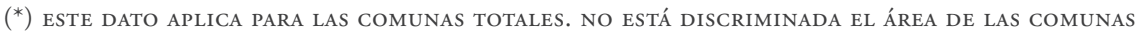
PERIFÉRICAS QUE ESTÁN POR FUERA DE LA CONCESIÓN DE AGUAS ANDINAS

FUENTE ELABORACIÓN PROPIA CON DATOS DE LA SUPERINTENDENCIA DE SERVICIOS SANITARIOS (SISS) Y DE LA ENCUESTA DE CARACTERIZACIÓN SOCIOECONÓMICA (CASEN) 2006 
Existe un grupo que lidera el consumo promedio por cliente en el área de concesión, con un consumo promedio que oscila entre $24,52 \mathrm{~m}^{3}$ y $41,77 \mathrm{~m}^{3}$, grupo en el cual existen dos tipos de comunas: por un lado, Providencia $\left(30,56 \mathrm{~m}^{3}\right)$ y Las Condes $\left(29,09 \mathrm{~m}^{3}\right)$, que están entre aquellas que tienen un tamaño promedio de hogar más pequeńo, pero en términos de ingresos son del llamado "cono de alta renta"; y por otro, las comunas de La Reina $\left(41,77 \mathrm{~m}^{3}\right)$, Peñalolén $\left(29,50 \mathrm{~m}^{3}\right)$ y Huechuraba $\left(28,95 \mathrm{~m}^{3}\right)$, que -a diferencia de las primeras- tienen un tamaño promedio de hogar alto, pero son también comunas donde habitualmente se localizan familias de altos ingresos, y por lo general en soluciones habitacionales unifamiliares de muy bajo coeficiente de ocupación predial.

El otro extremo del ranking, en términos de menores consumos promedio por cliente, lo ocupan comunas como Pudahuel $\left(16,39 \mathrm{~m}^{3}\right)$, La Pintana $\left(16,64 \mathrm{~m}^{3}\right)$ y Renca $\left(16,71 \mathrm{~m}^{3}\right)$; los tres casos corresponden a comunas con un tamaño promedio de hogar alto y localizadas en la periferia sur y occidental de menor nivel de ingreso autónomo promedio por hogar.

Al trazar una trayectoria en el tiempo para los periodos febrero 2009, febrero 2007 y febrero 2005, relativa al consumo comunal promedio por cliente del área de estudio, se demuestra que, salvo en cuatro comunas, en toda el área de la concesión, el consumo de cada comuna (32) decreció en el periodo 2005-2009: el promedio de consumo para todo el territorio operacional de Aguas Andinas se contrajo en 1,61 $\mathrm{m}^{3}$ por cliente al mes, partiendo de 22,69 $\mathrm{m}^{3}$ en 2005, y llegando a $21.08 \mathrm{~m}^{3}$ en 2009 .

Los consumos que están por encima del promedio de la concesión corresponden a once comunas, y se puede afirmar que mayoritariamente son aquellas que se localizan en el oriente de la ciudad, de sur a norte. Además, en este grupo de comunas, según la encuesta Casen de distintos años, se encuentran los mayores promedios de ingresos autónomos por hogar.

Los clientes -en promedio comunal- que consumen por debajo del promedio del área de estudio tienen, como característica espacial, una localización en el poniente de la aglomeración urbana y, al igual que el grupo anterior, con una distribución indiscriminada norte-sur. $\mathrm{Y}$ en contraste con los altos consumos, en términos parciales, se localizan los menores promedios de ingresos autónomos por hogar.

En este punto del patrón de localización por ingresos resalta, por no cumplir esta regla, la comuna de Santiago, que si bien registra un bajo promedio de consumo de agua potable, presenta un ingreso promedio por hogar de $\$ 865.067$, el cual puede ser considerado como un nivel de ingresos medio-alto. En este mismo sentido, en el otro grupo resalta también la comuna de Peńalolén, la cual se ubica en el tercer puesto de mayor consumo de agua potable y, sin embargo, sus ingresos promedio son de $\$ 716.975$, que aunque no son los más bajos de la urbe, representan menos de la mitad de los de la comuna Providencia (\$1.625.851), que está en el segundo lugar de consumo. Sin embargo, en términos generales, se ve una correspondencia entre promedio de unidades físicas de agua potable consumidas y el nivel de ingresos de los hogares que en promedio componen las comunas analizadas.

En cuanto al volumen consumido que ha sido subsidiado por el presupuesto del Estado a través de su esquema de subsidios focalizados implementado en el sector sanitario, el estudio muestra que los metros cúbicos subsidiados, en términos generales, 
son entregados de forma equivalente a las comunas que menor consumo registran. Destaca al respecto la comuna de San Ramón, que no solamente tiene el mayor promedio de clientes con acceso al subsidio, sino también el mayor promedio en volumen de agua proporcionada por dicha ayuda estatal. En contraposición, Santiago, Providencia y Las Condes son las comunas que menos clientes registran como beneficiarios del subsidio, y también en términos de volumen de agua subsidiada.

En síntesis, se observa una congruencia sólida en que los subsidios estatales para el consumo de agua potable están siendo entregados mayoritariamente, en términos de cantidad de clientes y en volumen de agua, a las comunas que menor promedio de consumo describieron para febrero de 2009.

\section{Montos facturados}

De la misma forma como se analizó el consumo promedio de unidades físicas de agua potable por cliente, en este apartado se va a tomar en consideración la combinación de ese consumo con el sistema tarifario legal vigente en la normativa sanitaria chilena (Figura 4).

FIgURA 4 Promedio monto total a pagar por cliente por comuna en el territorio operacional de Aguas Andinas (2009)

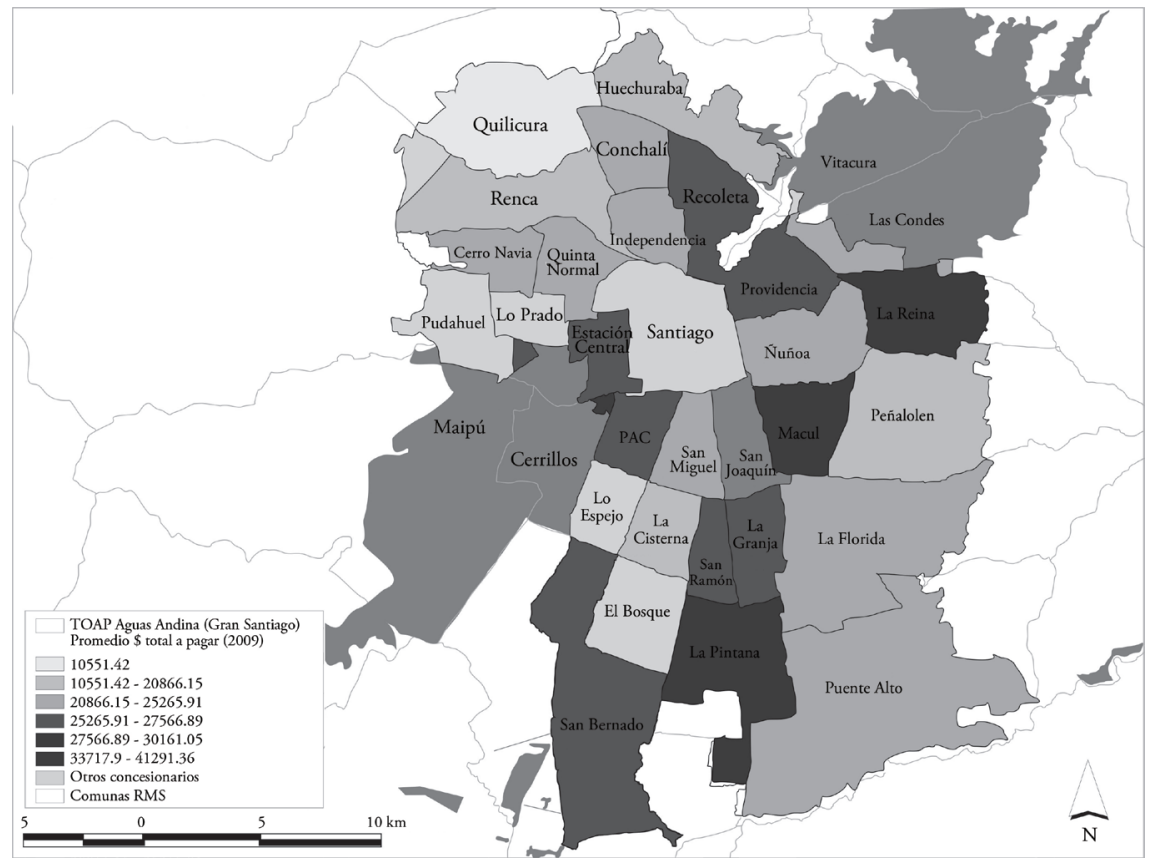

FUENTE ELABORACIÓN PROPIA CON DATOS DE LA SUPERINTENDENCIA DE SERVICIOS SANITARIOS (SISS)

En cuanto a los promedios comunales del monto total que debieron pagar los clientes de la concesionaria Aguas Andinas por concepto de su consumo durante el mes de febrero de 2009, se puede destacar que, en los tres primeros lugares de 
máxima facturación promedio por comuna, se encuentran la comuna de La Reina $(\$ 41.291,36)$, seguida por La Pintana $(\$ 38.717,22)$ y San Joaquín $(\$ 39.122,33)$, además del pequeño sector de Cerrillos $(\$ 36.649,10)$, que pertenece al área de concesión en estudio (Cuadro 2).

En el otro extremo de esta clasificación se encuentran tres comunas con los promedios más bajos de facturación: Santiago $(\$ 17.391,76)$, Lo Prado $(\$ 19.445,82)$ y Pudahuel $(\$ 20.866,15)$; además de un grupo muy pequeño de clientes que registran las bases de datos pertenecientes a la comuna de Maipú $(\$ 10.551,42)$.

Como se puede observar, el promedio general de facturación del territorio operacional de Aguas Andinas, que para febrero de 2009 fue de \$26.334,66 mensuales por cliente, al desagregarlo por comunas, tiene un amplio rango que se inicia en $\$ 10.551,42$, y termina en $\$ 41.291,36$.

CUADro 2 Promedios comunales del monto total de la facturación por cliente de la concesionaria Aguas Andinas, Región Metropolitana de Santiago, febrero 2009, 2007 y 2005, y porcentaje del monto total (2007) en ingreso autónomo promedio del hogar (2006)

\begin{tabular}{|c|c|c|c|c|}
\hline COMUNA & $\begin{array}{l}\text { MONTO TOTAL } \\
\text { POR PAGAR. } \\
\text { FEBRERO 2OO9 } \\
\text { (\$ CHILENOS) }\end{array}$ & $\begin{array}{l}\text { MONTO TOTAL } \\
\text { POR PAGAR. } \\
\text { FEBRERO 20O7 } \\
\text { (\$ CHILENOS) }\end{array}$ & $\begin{array}{l}\text { MONTO TOTAL } \\
\text { POR PAGAR. } \\
\text { FEBRERO 2005 } \\
\text { (\$ CHILENOS) }\end{array}$ & $\begin{array}{c}\text { PORCENTAJE DEL } \\
\text { MONTO TOTAL } \\
\text { (FEB. 2007) EN EL } \\
\text { INGRESO AUTÓNOMO } \\
\text { PROMEDIO DEL } \\
\text { HOGAR }(2006)(\%)(*)\end{array}$ \\
\hline Maipú & $10.551,42$ & $5.384,05$ & $1.856,70$ & 0,70 \\
\hline Santiago & $17.391,76$ & $16.180,19$ & $16.470,18$ & 1,87 \\
\hline Lo Prado & $19.445,82$ & $16.172,27$ & $15.753,49$ & 3,21 \\
\hline Pudahuel & $20.866,15$ & $16.763,70$ & $16.006,77$ & 2,83 \\
\hline Macul & $22.641,91$ & $17.499,75$ & $16.360,78$ & 3,08 \\
\hline San Miguel & $23.300,61$ & $19.140,37$ & $18.492,85$ & 1,60 \\
\hline Las Condes & $24.167,89$ & $19.542,54$ & $18.986,04$ & 0,96 \\
\hline Conchalí & $24.371,03$ & $21.523,34$ & $20.647,25$ & 4,13 \\
\hline La Florida & $24.498,93$ & $18.230,76$ & $17.109,94$ & 2,03 \\
\hline Independencia & $24.510,83$ & $20.527,31$ & $21.480,90$ & 3,36 \\
\hline Quinta Normal & $24.641,01$ & $20.572,46$ & $20.317,02$ & 3,83 \\
\hline Cerro Navia & $24.956,95$ & $21.991,35$ & $20.620,15$ & 5,39 \\
\hline La Cisterna & $24.994,71$ & $19.724,00$ & $18.547,76$ & 2,71 \\
\hline Ñuñoa & $25.008,58$ & $20.376,62$ & $19.897,49$ & 1,42 \\
\hline Puente Alto & $25.265,91$ & $17.376,81$ & $14.221,47$ & 2,47 \\
\hline Providencia & $25.784,38$ & $21.609,54$ & $21.395,68$ & 1,33 \\
\hline La Granja & $26.086,67$ & $18.794,95$ & $18.330,10$ & 4,30 \\
\hline San Bernardo & $26.170,45$ & $19.106,72$ & $18.127,40$ & 3,99 \\
\hline Estación Central & $26.337,49$ & $22.543,09$ & $21.689,70$ & 3,48 \\
\hline
\end{tabular}


(continuación)

\begin{tabular}{|c|c|c|c|c|}
\hline COMUNA & $\begin{array}{l}\text { MONTO TOTAL } \\
\text { POR PAGAR. } \\
\text { FEBRERO } 2009 \\
\text { (\$ CHILENOS) }\end{array}$ & $\begin{array}{l}\text { MONTO TOTAL } \\
\text { POR PAGAR. } \\
\text { FEBRERO 2007 } \\
\text { (\$ CHILENOS) }\end{array}$ & $\begin{array}{l}\text { MONTO TOTAL } \\
\text { POR PAGAR. } \\
\text { FEBRERO 2OO5 } \\
\text { (\$ CHILENOS) }\end{array}$ & $\begin{array}{c}\text { PORCENTAJE DEL } \\
\text { MONTO TOTAL } \\
\text { (FEB. 2007) EN EL } \\
\text { INGRESO AUTÓNOMO } \\
\text { PROMEDIO DEL } \\
\text { HOGAR }(2006)(\%)(*)\end{array}$ \\
\hline San Ramón & $26.640,65$ & $19.992,62$ & $17.194,58$ & 3,49 \\
\hline Recoleta & $27.023,85$ & $23.408,87$ & $24.159,54$ & 5,05 \\
\hline Pedro Aguirre Cerda & $27.566,89$ & $23.886,17$ & $20.666,81$ & 4,40 \\
\hline El Bosque & $29.351,79$ & $23.083,73$ & $21.997,60$ & 4,97 \\
\hline Quilicura & $29.882,62$ & $21.254,37$ & $15.904,25$ & 3,28 \\
\hline Lo Espejo & $30.161,05$ & $24.109,47$ & $22.648,67$ & 5,82 \\
\hline Renca & $31.654,58$ & $30.872,75$ & $31.508,66$ & 5,98 \\
\hline Peñalolén & $32.015,93$ & $22.905,71$ & $21.301,71$ & 3,19 \\
\hline Huechuraba & $33.717,90$ & $27.059,09$ & $29.584,98$ & 3,93 \\
\hline Cerrillos & $36.649,10$ & $25.348,37$ & $20.607,32$ & 3,80 \\
\hline La Pintana & $38.717,22$ & $31.557,64$ & $29.367,68$ & 7,82 \\
\hline San Joaquín & $39.122,33$ & $34.890,69$ & $30.224,32$ & 6,08 \\
\hline La Reina & $41.291,36$ & $30.879,11$ & $28.997,56$ & 2,42 \\
\hline Total & $26.334,66$ & $20.977,69$ & $19.665,80$ & \\
\hline
\end{tabular}

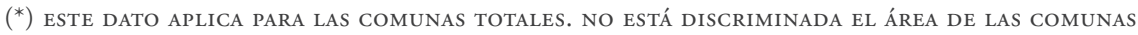
PERIFÉRICAS QUE ESTÁN POR FUERA DE LA CONCESIÓN DE AGUAS ANDINAS

FUENTE ELABORACIÓN PROPIA CON DATOS DE LA SUPERINTENDENCIA DE SERVICIOS SANITARIOS (SISS) Y DE LA ENCUESTA CASEN 2006

Para continuar el análisis, se incorporarán las trayectorias de tiempo febrero 2007 y febrero 2005, con las cuales se pueden hacer varias reflexiones. La primera de ellas es que, a diferencia del promedio de consumo de unidades físicas, donde cuatro comunas presentaban una tendencia contraria al promedio de decrecimiento en el consumo, en este campo de los montos totales por pagar, ninguno baja; todos (algunos en mayor y otros en menor proporción) tienen un incremento de su facturación durante el periodo febrero 2005 a febrero 2009 .

En promedio, para los clientes residenciales de Aguas Andinas, la evidencia muestra que sus cuentas entre 2005 y 2009 se incrementaron un 33,9\% en valores corrientes que, al ser corregidos por el IPC generado en ese periodo, se traduce en un $9,9 \%$ en valores reales.

De las catorce comunas que registraron una facturación por encima del promedio del área de concesiones, se pueden hacer dos grupos. El primero está compuesto por La Reina, Peñalolén, Huechuraba y Cerrillos, que conservan una congruencia en términos de que también sus comunas pertenecían a aquellas que estaban por encima del promedio de consumo de unidades físicas de agua potable en el mismo periodo (febrero 2009). En el segundo grupo de comunas -integrado por Estación Central, San Ramón, Recoleta, Pedro Aguirre Cerda, El Bosque, Quilicura, Lo Espejo, Renca, La Pintana y San Joaquín-, a pesar de haberse superado el promedio 
de facturación mensual en el área de estudio, no se constató lo mismo cuando se calculó el promedio de consumo de unidades físicas, de modo que se presenta una incongruencia en estos dos promedios.

Las dieciocho comunas que facturaron por debajo del promedio del territorio operacional de Aguas Andinas, al igual que las de facturación superior, se pueden dividir en dos grupos. Por una parte, las comunas de Pudahuel, Maipú, Santiago, Lo Prado, Cerro Navia, La Granja, Puente Alto, Conchalí, San Bernardo, Quinta Normal e Independencia, que muestran congruencia respecto a este dato y el promedio de consumo de metros cúbicos registrado en el mismo periodo de estudio. Por otra, están aquellas comunas que, en cuanto a su consumo físico de agua, formaban parte del grupo que está por encima del promedio de la concesión, a saber, Macul, San Miguel, Las Condes, La Florida, La Cisterna, Nuuñoa y Providencia; sin embargo, en esta valoración de la facturación mensual, dichas comunas están por debajo del promedio de Aguas Andinas.

El grupo que supera el promedio de facturación en la concesión, a diferencia del patrón espacial identificado a partir de los promedios de consumo de unidades físicas, en este caso es menos claro. Aunque en términos muy generales conserva la misma distribución, esta se hace un poco más dispersa, al incluir comunas como Renca y Quilicura, que antes ayudaban a conformar un grupo concentrado de bajos consumos al poniente de la ciudad, pero que, en relación con su facturación, pasaron a integrar el grupo superior junto a otras comunas del oriente santiaguino.

Lo mismo ocurre en términos espaciales con aquellas comunas de altos promedios de consumo, que marcaban un patrón de localización en el costado oriental de la ciudad. Aunque casi todas las comunas de esos cuadrantes ahora pertenecen al grupo de baja facturación, comunas como La Reina y Peñalolén lideran, a su vez, los promedios de la más alta facturación en el territorio operacional de Aguas Andinas.

Un dato adicional, que resulta muy iluminador en este ejercicio de analizar los promedios comunales de facturación al interior de la concesión en estudio, es qué porcentaje (aproximado) del ingreso promedio de los hogares de la comuna supone el pago mensual de los servicios prestados por Aguas Andinas. Para el conjunto de las comunas estudiadas ${ }^{2}$, el rango de este porcentaje oscila entre el 0,70\% y 7,82\% de los ingresos promedio respectivos de las comunas. En síntesis, para los clientes de la comuna que marca el piso del rango, pagar el servicio de agua potable y alcantarillado representa menos del 1\% de sus ingresos; y en contraste, la comuna que registra el techo del rango paga proporcionalmente ocho veces más por el mismo servicio.

Analizando los extremos de la distribución de este dato, el primer grupo puede contener a aquellas comunas a las que, en promedio, el monto total facturado por Aguas Andinas representa menos del 2\% de los ingresos comunales promedio por hogar: Maipú $(0,70 \%)$, Las Condes $(0,96 \%)$, Providencia $(1,33 \%)$, Nuñoa $(1,42 \%)$, San Miguel (1,60\%) y Santiago (1,87\%).

Descontando el caso de Maipú, que es una proporción muy pequeña de clientes en la comuna, y Santiago, que tiene un tamaño promedio de hogar muy pequeño,

2 Este dato es una aproximación construida a partir de la relación de datos de consumo promedio en febrero de 2007, y datos comunales agregados de ingreso autónomo por hogar de la encuesta Casen 2006; como lo indica la Tabla 6. 
las otras cuatro comunas conforman un grupo que comparte tres características: a) están dentro de los altos consumidores de agua potable en promedio de volumen de la concesión (con excepción de la comuna de Santiago); b) registran también los tamaños promedio de hogar más pequeños; y c) en términos comparativos, el monto total de la facturación por el agua que consumen es menor al promedio de área de estudio y así, en relación con los ingresos de los hogares y en términos proporcionales, el servicio les resulta mucho más económico que al resto de la ciudad.

El segundo grupo contiene a las comunas a las que, en promedio, cancelar el monto facturado de agua potable les significa disponer de más del $5 \%$ de los ingresos percibidos por los hogares: Recoleta (5,05\%), Cerro Navia (5,39\%), Lo Espejo (5,82\%), Renca (5,98\%), San Joaquín (6,08\%) y, el mayor de todos, La Pintana $(7,82 \%)$.

En términos casi absolutos, estas seis comunas comparten tres características: a) todas ellas están por debajo del promedio de consumo de unidades físicas de agua potable en la concesión y, en contraste, cinco de ellas (exceptuando Cerro Navia) se ubican por encima del promedio del monto total a pagar por el servicio; b) excluyendo las comunas de Recoleta y San Joaquín, representan un grupo de comunas con un tamaño promedio de hogar superior en comparación con las otras comunas del área de estudio; y c) son comunas donde, en relación con sus ingresos promedio, el agua les resulta mucho más costosa que al resto de Santiago, en términos proporcionales.

Un punto aparte en la reflexión merece la asignación del subsidio estatal como promedio del monto total entregado por comuna. Este indicador es liderado en solitario por la comuna de San Ramón, con un monto promedio subsidiado de $\$ 1.092,47$ por cliente, seguida por las comunas de Cerro Navia, Lo Prado, Macul y La Granja, cuyo monto promedio oscila entre \$606,68 y \$783,02.

En términos generales, se observa que las cinco comunas mencionadas no responden en rigor a las variables de análisis utilizadas en este capítulo, pues si se observa el tamaño promedio por hogar en ellas y el porcentaje de sus ingresos que ocupan para pagar el monto de facturación, no existen equivalencias.

En cuanto al volumen de agua consumida en unidades físicas, y a la facturación producto de este consumo, se puede afirmar que estas cinco comunas que acceden en términos monetarios a mejores subsidios, se focalizan con una débil tendencia hacia los consumidores pequeños, o que están por debajo del promedio de consumo del área de concesión de Aguas Andinas.

\section{Conclusiones}

Este trabajo demuestra que los avances en eficiencia y mejoramiento integral del servicio sanitario a partir del proceso de privatización han tenido impactos positivos en términos ambientales, pues han producido un sistema que demanda menos agua de las fuentes naturales (y, por lo tanto, contamina menos). No obstante, tales avances no han sido capaces de reducir la brecha de consumo ni las inequidades en la producción de agua por habitante entre las empresas que operan en la Región Metropolitana de Santiago. Es así que los altos consumidores de agua 
potable de la concesión de Aguas Andinas registran también los promedios de hogar más pequeños. Además, en términos comparativos, son aquellos a quienes -considerando sus ingresos- el servicio les resulta mucho más económico, en comparación con el resto de la ciudad, en términos proporcionales. Por otra parte, los clientes que están por debajo del promedio de consumo de unidades físicas de agua potable en la concesión, pertenecen a un grupo de comunas con un tamańo promedio de hogar superior al del resto del área de estudio, además de ser comunas donde, respecto de los ingresos promedio de los hogares, el agua les resulta mucho más costosa que al resto de Santiago, en términos proporcionales.

Por consiguiente, el ingreso y su capacidad de constituir el proyecto familiar, sin duda alguna, es el factor determinante en la construcción del concepto de nueva pobreza urbana, pues aparentemente la ciudad capitalista resolvió el acceso al agua en términos estructurales (distribución y recolección), pero no en términos de equidad. No obstante, profundizar en el ámbito del consumo, y lo que esto representa para una familia pobre, puede ser otra clave para comprender nuevas formas de exclusión en la ciudad.

En síntesis, este trabajo, por una parte, puso de relieve el indicador del consumo como un instrumento para la visualización de nuevas manifestaciones de la pobreza urbana en un sector donde se ha alcanzado la universalización del acceso al servicio del agua. Y por otra, desde una perspectiva empírica, constató que la contracción del consumo domiciliario de agua potable que vienen registrando los sectores de más bajos ingresos a partir del proceso de privatización, responde a la configuración de un sistema tarifario en permanente proceso de encarecimiento y a su desbalance respecto a un ingreso familiar precario, reforzando así los mecanismos generadores de desequilibrios territoriales preexistentes en la ciudad de Santiago.

\section{Referencias bibliográficas}

Bakker, K. (2001). Paying for water: Water charging and equity in England and Wales. Transactions of the Institute of British Geographers, New Series, 26(2), 143-164.

Bauer, C.J. (2002). Contra la corriente: Privatización, mercados de agua y el Estado en Chile. Ecología y medio ambiente. Santiago: Lom Ediciones.

Davis, J. (2005). Private-sector participation in the water and sanitation sector. Annual Review of Environment and Resources, 30, 145-83. doi: 10.1146/annurev. energy.30.050504.144635

Figueroa, O. (2004). Infraestructura, servicios públicos y expansión urbana en Santiago. En C. De Mattos, M.E. Ducci, A. Rodríguez, G. Yáñez Warner (Eds.), Santiago en la globalización: ¿Una nueva ciudad? (pp. 243-272). Santiago: Ediciones SUR-EURE Libros.

Jorgensen, B., Graymore, B. \& O'Toole, K. (2009). Household water use behavior: An integrated model. Journal of Environmental Management, 91(1), 227-236. doi:10.1016/j.jenvman.2009.08.009

Kayaga, S., Calvert, J. \& Sansom, K. (2003). Paying for water services: Effects of household characteristics. Utilities Policy, 11(3), 123-132. doi:10.1016/S0957-1787(03)00034-1 
Morandé, F. \& Doña, J. (1997). Los servicios de agua potable en Chile: Condicionantes, institucionalidad y aspectos de economía politica. Washington, DC: Banco Interamericano de Desarrollo (BID). Disponible en http://www.iadb.org/res/laresnetwork/files/ pr15finaldraft.pdf

Pflieger, G. (2008). Historia de la universalización del acceso al agua y alcantarillado en Santiago de Chile (1970-1995). EURE, 34(103), 131-152. http://dx.doi.org/10.4067/S025071612008000300007

Rodríguez, A. (1994). Cuatro historias de servicios urbanos en América Latina y una explicación. En A. Puncel Chornet (Ed.), Las ciudades de América Latina: Problemas y oportunidades (pp. 25-34). Valencia: Col-lecció Oberta, Universitat de València.

siss (Superintendencia de Servicios Sanitarios). Informes anuales de gestión. De 1999 a 2007. Disponibles en http://www.siss.cl/577/w3-propertyvalue-3443.html

Swyngedouw, E. (2004). Social power and the urbanization of water: flows of power. Oxford, R.U.: Oxford University Press.

Tironi Rodó, M. (2003). Nueva pobreza urbana: Vivienda y capital social en Santiago de Chile, 1985-2001. Santiago: Universidad de Chile/Ril Editores.

Valenzuela, S. \& Jouravlev, A. (2007). Servicios urbanos de agua potable y alcantarillado en Chile: Factores determinantes del desempeño. Serie Recursos Naturales e Infraestructura, 123. Santiago: Comisión Económica para América Latina y el Caribe (CEPAL). Disponible en http://repositorio.cepal.org/bitstream/handle/11362/6321/S0700281_ es.pdf?sequence $=1$

Wilson, W.J. (1998). When work disappears: new implications for race and urban poverty in the global economy. CASE paper 17. Centre for Analysis of Social Exclusion, London School of Economics. Disponible en http://library.bsl.org.au/jspui/bitstream/1/294/1/ CASEpaper\%20no17.pdf

Winpenny, J. (1994). Managing water as an economic resource. Londres: Routledge.

Zaki, S. \& Amin, N. (2009). Does basic services privatization benefit the urban poor? Some evidence from water supply privatization in Thailand. Urban Studies, 46(11), 2301-2327.

Zhanga, H. \& Brownb, D. (2005). Understanding urban residential water use in Beijing and Tianjin, China. Habitat International, (29) 469-491. doi:10.1016/j.habitatint.2004.04.002 